PROCEEDINGS OF THE

AMERICAN MATHEMATICAL SOCIETY

Volume 137, Number 9, September 2009, Pages 2825-2833

S 0002-9939(09)09902-X

Article electronically published on April 23, 2009

\title{
BETTI NUMBER BOUNDS FOR FEWNOMIAL HYPERSURFACES VIA STRATIFIED MORSE THEORY
}

\author{
FRÉDÉRIC BIHAN AND FRANK SOTTILE
}

(Communicated by Daniel Ruberman)

\begin{abstract}
We use stratified Morse theory for a manifold with corners to give a new bound for the sum of the Betti numbers of a fewnomial hypersurface in $\mathbb{R}_{>}^{N}$
\end{abstract}

In the book Fewnomials [6, A. Khovanskii gives bounds on the Betti numbers of varieties $X$ in $\mathbb{R}^{n}$ or in the positive orthant $\mathbb{R}_{>}^{n}$. These varieties include algebraic varieties, varieties defined by polynomial functions in the variables and exponentials of the variables, and also by even more general functions. Here, we consider only algebraic varieties. For these, Khovanskii bounds the sum $b_{*}(X)$ of Betti numbers of $X$ by a function that depends on $n$, the codimension of $X$, and the total number of monomials appearing (with nonzero coefficients) in polynomial equations defining $X$.

For real algebraic varieties $X$, the problem of bounding $b_{*}(X)$ has a long history. O. A. Olĕnik [8] and J. Milnor [7] used Morse theory to estimate the number of critical points of a suitable Morse function to obtain a bound. R. Thom 12 used Smith Theory to bound the mod-2 Betti numbers. This Smith-Thom bound has the form $b_{*}(X) \leq b_{*}\left(X_{\mathbb{C}}\right)$, where $X_{\mathbb{C}}$ is the complex variety defined by the same polynomials as $X$. For instance, if $X \subset \mathbb{R}^{n}$ is defined by polynomials of degree at most $d$, then Milnor's bound is $b_{*}(X) \leq d(2 d-1)^{n}$. If $X \subset(\mathbb{R} \backslash\{0\})^{n}$ is a smooth hypersurface defined by a polynomial with Newton polytope $P$, then the Smith-Thom bound is $b_{*}(X) \leq b_{*}\left(X_{\mathbb{C}}\right)=n ! \cdot \operatorname{Vol}(P)$, where $\operatorname{Vol}(P)$ is the usual volume of $P$. We refer to [10] for an informative history of the subject and to the book [1] for more details.

These bounds are given in term of degree or volume of a Newton polytope, which is a numerical deformation invariant of the complex variety $X_{\mathbb{C}}$. In contrast, the topology of a real algebraic variety depends on the coefficients of its defining equations, and in particular, on the number of monomials involved in these equations. For instance, Descartes's rule of signs implies that the number of positive roots of a real univariate polynomial is less than its number of monomials, but the number

Received by the editors June 19, 2008.

2000 Mathematics Subject Classification. Primary 14P25.

Key words and phrases. Stratified Morse theory, fewnomials, Betti numbers.

The second author was supported by NSF CAREER grant DMS-0538734 and NSF grant DMS0701050 .

(C)2009 American Mathematical Society Reverts to public domain 28 years from publication 
of complex roots is equal to its degree. Khovanskii's bound can be seen as a generalization of this Descartes bound. It is smaller than the previous bounds when the defining equations have few monomials compared to their degrees. While it has always been clear that Khovanskii's bounds are unrealistically large, it appears very challenging to sharpen them. Some progress has been made recently for the number of nondegenerate positive solutions to a system of $n$ polynomials in $n$ variables [3]. Here, we bound $b_{*}(X)$, when $X$ is a fewnomial hypersurface.

Suppose that $X \subset \mathbb{R}_{>}^{n}$ is a smooth hypersurface defined by a Laurent polynomial with $n+l+1$ distinct monomial terms. Khovanskii [6] (Corollary 4, p. 91) showed that

$$
b_{*}(X) \leq\left(2 n^{2}-n+1\right)^{n+l}(2 n)^{n-1} 2^{\left(\begin{array}{c}
n+l \\
2
\end{array}\right)} .
$$

We use the new upper bound [3] of $\frac{e^{2}+3}{4} 2^{\left(\begin{array}{l}l \\ 2\end{array}\right)} n^{l}$ on the number of nondegenerate positive solutions to a system of $n$ polynomials in $n$ variables having $n+l+1$ monomial terms, together with stratified Morse theory for a manifold with corners [5] to give a new bound for the sum $b_{*}(X)$ of the Betti numbers of a fewnomial hypersurface. Fix positive integers $N \geq n$ and $l$.

Theorem 1. Let $X$ be a hypersurface in $\mathbb{R}_{>}^{N}$ defined by a polynomial with $n+l+1$ monomials whose exponent vectors have affine span of dimension $n$. Then

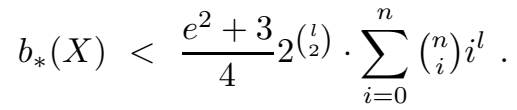

The bound of Theorem 1 is bounded above by the simpler expression

$$
\left(e^{2}+3\right) 2^{\left(\begin{array}{l}
l \\
2
\end{array}\right)} n^{l} \cdot 2^{n-3},
$$

which is smaller than the bound on the number of connected components (zeroth Betti number) proven in 2 .

The idea of the proof is as follows. If $X \subset \mathbb{R}_{>}^{n}$ is compact, then a generic monomial function will be a Morse function on $X$. The critical points of this Morse function are positive solutions to a polynomial system with the same initial monomials and we may apply the bound $\underline{3}$. If $X$ is noncompact, consider its image $Z=\log (X)$ under the coordinatewise logarithmic map (a homeomorphism) and take the intersection with a sufficiently large simplex $\Delta$ so that $b_{*}(Z)=b_{*}(Z \cap \Delta)$. The intersection of $Z$ with faces of $\Delta$ are parts of fewnomial hypersurfaces about which we have a control on the number of variables and monomials. The singular space $Z \cap \Delta$ is stratified by these hypersurfaces in the faces of $\Delta$. In fact $Z \cap \Delta$ is a manifold with corners.

Stratified Morse theory as developed by Goresky and MacPherson extends classical Morse theory to compact stratified spaces. While it is complicated to apply this theory in general, it is quite simple for manifolds with corners. A stratified Morse function is a function whose restrictions to strata are usual Morse functions. By stratified Morse theory, $b_{*}(Z \cap \Delta)$ is bounded from above by the total number of critical points of the restrictions to the strata of a stratified Morse function. We use a generic linear function as a stratified Morse function on $Z \cap \Delta$. Such a linear function comes from a monomial function on $X$, and the number of critical points for each stratum may be estimated by the bound $[3$ as in the compact case.

Khovanskii's bound (1) is a special case of more general bounds which he obtains for fewnomial complete intersections. It remains an important open problem to 
adapt the methods of 3 ] to fewnomial complete intersections. We remark that our results hold for polynomials with real-number exponents. In fact, our proofs (and the proofs in [3]) involve this added generality. We first describe Morse theory for a manifold with corners in Section 1 and prove Theorem 1 in Section 2.

We would like to thank Jean-Jacques Risler for providing us with useful historical references on the subject.

\section{Morse THEORY FOR A MANIFOLD WITH CORNERS}

In classical Morse theory, the topology of a compact differentiable manifold $X$ is inferred from critical points of a sufficiently general smooth function $f: X \rightarrow \mathbb{R}$, called a Morse function. For example, $b_{*}(X)$ is bounded above by the number of critical points of any Morse function. Goresky and MacPherson [5] develop a version of Morse theory for stratified spaces. This is particularly simple for manifolds with corners, which for us will be the intersection of our fewnomial hypersurface with a large simplex whose every face meets it transversally.

We begin with sketches of classical and of stratified Morse theory, and then explain how stratified Morse theory applies to a manifold with corners. For further discussion and proofs see [5].

A smooth function $f: X \rightarrow \mathbb{R}$ on a compact differential manifold is a Morse function if its critical values (in $\mathbb{R}$ ) are distinct and each critical point (in $X$ ) of $f$ is nondegenerate (the Hessian matrix of second partial derivatives has full rank). This implies that the critical points are discrete and there are finitely many of them. For each $c \in \mathbb{R}$, set $X_{c}:=f^{-1}(-\infty, c]$. If $c$ is smaller than any critical value, then $X_{c}$ is empty, and if $c$ is greater than all critical values, then $X=X_{c}$. The Morse Lemmata describe how the topological type of $X_{c}$ changes as $c$ increases from $-\infty$ to $\infty$. The first Morse Lemma asserts that the topological type of $X_{c}$ is constant for all $c$ lying in an open interval that contains no critical values. The second Morse Lemma asserts that if $(a, b)$ contains a unique critical value $c=f(p)$, then the pair $\left(X_{b}, X_{a}\right)$ is homeomorphic to the pair $\left(D^{\lambda} \times D^{n-\lambda},\left(\partial D^{\lambda}\right) \times D^{n-\lambda}\right)$. Here, $X$ has dimension $n, D^{m}$ is a closed disc of dimension $m$, and $\lambda$ is the number of negative eigenvalues of the Hessian matrix of $f$ at $p$. The long exact sequence of a pair and induction on the critical values $c$ shows that the sum of the Betti numbers of $X$ is bounded above by the number of critical points of $f$.

Suppose now that $X$ is a Whitney stratified space, which we assume is embedded in an ambient manifold $W$. A smooth function $f: X \rightarrow \mathbb{R}$ is the restriction to $X$ of a smooth function on $W$. A critical point of $f$ is a critical point of the restriction of $f$ to any stratum. (Each stratum in a Whitney stratified space is a manifold.) A Morse function $f: X \rightarrow \mathbb{R}$ is a smooth function on $X$ whose critical values are distinct, and at each critical point $p$ of $F$, the restriction of $f$ to the stratum containing $p$ is nondegenerate at $p$. There is a third condition that the differential of $f$ at $p$ does not annihilate any limit of tangent spaces to any stratum other than the stratum containing $p$.

In stratified Morse theory, the first Morse Lemma holds as before and the second Morse Lemma is modified as follows. Let $p$ be a critical point of $f$ lying in a stratum $S$ of $X$. Then let $D(p)$ be a small disk in $W$ transverse to the stratum $S$ such that $D(p) \cap S=\{p\}$ and call its intersection $N(p)$ with $X$ the normal slice to $S$ at $p$. Normal Morse data at $p$ are a pair $(A, B)$, where $A$ is the set of points $x$ in $N(p)$ for which $|f(x)-f(p)| \leq \epsilon$ and $B$ is that part of the boundary of $A$ where 
$f(x)=f(p)-\epsilon$, and $\epsilon$ is any sufficiently small positive number. The tangential Morse data at $p$ are the pair $\left(D^{\lambda} \times D^{n-\lambda},\left(\partial D^{\lambda}\right) \times D^{n-\lambda}\right)$ appearing in the second Morse Lemma for the Morse function $f$ restricted to the stratum $S$, which is a manifold of dimension $n$. The second Morse Lemma in stratified Morse theory asserts that if the interval $(a, b)$ contains a unique critical value $c=f(p)$, then the pair $\left(X_{b}, X_{a}\right)$ is homeomorphic to the product of pairs

$$
\text { (normal Morse data at } p) \times(\text { tangential Morse data at } p) \text {. }
$$

(Recall that the product $(A, B) \times\left(A^{\prime}, B^{\prime}\right)$ of pairs is the pair $\left(A \times A^{\prime}, A \times B^{\prime} \cup A^{\prime} \times\right.$ $B)$.) In general, we must have detailed information about the interaction between the Morse function and the stratification to use stratified Morse theory.

Such detailed information is available for manifolds with corners. Let $\mathbb{R}_{\geq}:=$ $[0, \infty)$ be the nonnegative real numbers and $\mathbb{R}_{\geq}^{m}$ be the nonnegative orthant in $\mathbb{R}^{m}$. A manifold with corners is a compact topological space $X$ with a covering by charts, each homeomorphic to $\mathbb{R}_{\geq}^{m} \times \mathbb{R}^{n}$, where $m+n$ is the dimension of $X$. Each point of $X$ has a well-defined tangent space isomorphic to $\mathbb{R}^{m+n}$, and has a tangent cone isomorphic to $\mathbb{R}_{\geq}^{m} \times \mathbb{R}^{n}$. Points with tangent cone isomorphic to $\mathbb{R}_{\geq}^{m} \times \mathbb{R}^{n}$ for $m$ fixed form a submanifold of dimension $n$. All such submanifolds form the boundary strata of $X$.

Suppose that $f: X \rightarrow \mathbb{R}$ is a Morse function. Let $p$ be a point in a boundary stratum locally homeomorphic to $\mathbb{R}_{\geq}^{m} \times \mathbb{R}^{n}$. The normal slice $N(p)$ to such a point is a neighborhood of the origin in the cone $\mathbb{R}_{\geq}^{m}$. Due to the third condition on Morse functions, there are exactly two possibilities for the normal Morse data $(A, B)$ at $p$. The first component $A$ is homeomorphic to the $m$-simplex

$$
\Delta_{m}:=\left\{x \in \mathbb{R}_{\geq}^{m}:|x|:=x_{1}+\cdots+x_{m} \leq 1\right\} \quad\left(\simeq D^{m}\right)
$$

and the second component $B$ is either the empty set $\emptyset$ (if $f(p)$ is locally the minimum value of $f$ on $N(p)$ ) or a contractible subset of the (m-1)-simplex $|x|=1$ otherwise. (If $f(p)$ is a local maximum, then $B$ is the full $(m-1)$-simplex.) We illustrate this in Figure 1
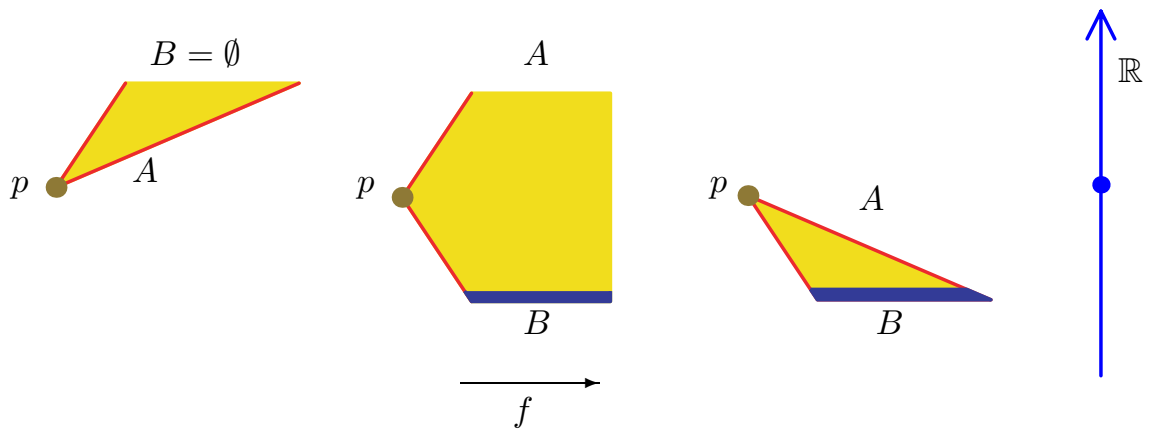

Figure 1. Normal Morse data

Observe that in the case when $B=\emptyset$, we have

$$
(A, B) \times\left(D^{\lambda} \times D^{n-\lambda},\left(\partial D^{\lambda}\right) \times D^{n-\lambda}\right) \simeq\left(D^{\lambda} \times D^{m+n-\lambda},\left(\partial D^{\lambda}\right) \times D^{m+n-\lambda}\right) .
$$

Thus this critical point could contribute to the sum of the Betti numbers of $X$. On the other hand, when $B \neq \emptyset$, the pair $(B, B)$ is a deformation retract of $(A, B)$, 
and so the critical point $p$ does not contribute to the sum of the Betti numbers of $X$, as the topology of $X_{a}$ does not change as $a$ passes $f(p)$.

Proposition 2. The sum of the Betti numbers of a manifold $Z$ with corners is at most the number of critical points $p$ of a Morse function $f$ for $Z$ where the minimum of $f$ on the normal slice to $Z$ at $p$ is attained at $p$.

Example 3. Proposition 2 is illustrated by the cannoli shell, which is a manifold with corners. It is a cylinder $D^{1} \times S^{1}$ having two boundary strata, each homeomorphic to the circle $S^{1}$. The height function of Figure 2 is a Morse function for the cannoli shell with four critical points. Only the two smallest critical values contribute to its topology and its Betti numbers, by Proposition 2 ,

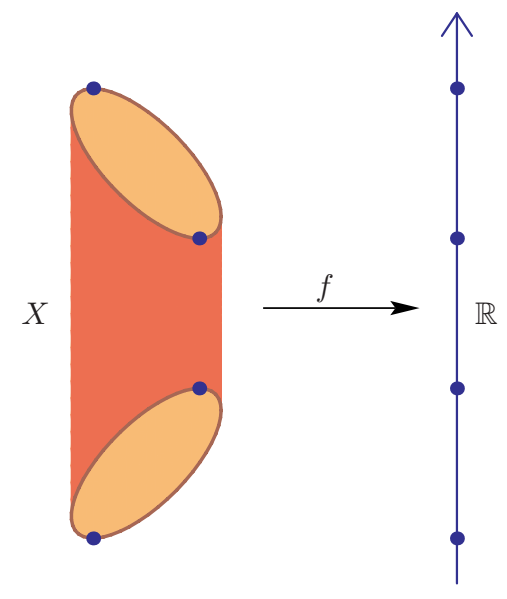

FiguRE 2. Morse function on the cannoli shell

\section{Proof of Theorem 1}

Let $f\left(x_{1}, \ldots, x_{N}\right)$ be a real Laurent polynomial with $n+l+1$ exponent vectors that span an $n$-dimensional affine subspace of $\mathbb{R}^{N}$ such that $X:=\mathcal{V}(f) \subset \mathbb{R}_{>}^{N}$ is a smooth hypersurface. Rather than work with polynomials in $\mathbb{R}_{>}^{N}$, we work with exponential sums in $\mathbb{R}^{N}$. These notions are related via a logarithmic change of coordinates. Consider the isomorphisms of Lie groups

$$
\begin{aligned}
& \operatorname{Exp}: \mathbb{R}^{N} \longrightarrow \mathbb{R}_{>}^{N} \quad \log : \mathbb{R}_{>}^{N} \longrightarrow \mathbb{R}^{N} \\
& \left(z_{1}, \ldots, z_{N}\right) \longmapsto\left(e^{z_{1}}, \ldots, e^{z_{N}}\right) \quad\left(x_{1}, \ldots, x_{N}\right) \longmapsto\left(\ln \left(x_{1}\right), \ldots, \ln \left(x_{N}\right)\right) .
\end{aligned}
$$

Under this isomorphism monomials $x^{\alpha}$ correspond to exponentials $e^{z \cdot \alpha}$, and so the fewnomial $f=\sum_{i} c_{i} x^{\alpha_{i}}$ corresponds to the exponential sum

$$
\varphi:=\sum_{i=0}^{n+l} c_{i} e^{z \cdot \alpha_{i}}
$$

Let $Z:=\mathcal{V}(\varphi) \subset \mathbb{R}^{N}$ be the hypersurface defined by $\varphi$, which is homeomorphic to $X$. For exponential sums, it is quite natural to allow the exponents $\alpha_{i}$ to be real vectors. We will prove Theorem 1 in these logarithmic coordinates and with real exponents. 
Theorem 1. The sum of the Betti numbers of a hypersurface in $\mathbb{R}^{N}$ defined by an exponential sum with $n+l+1$ terms whose exponent vectors have affine span of dimension $n$ is at most

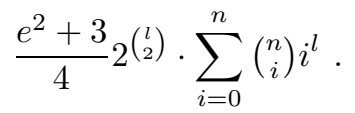

Multiplying $\varphi$ by $e^{-z \cdot \alpha_{0}}$, we may assume that $\alpha_{0}=0$. Then $\alpha_{1}, \ldots, \alpha_{n+l}$ span an $n$-dimensional linear subspace of $\mathbb{R}^{N}$. After a linear change of coordinates, we may assume that $\varphi$ only involves the first $n$ variables, and thus the hypersurface $Z$ becomes a cylinder

$$
Z \simeq\left\{z \in \mathbb{R}^{n}: \varphi(z)=0\right\} \times \mathbb{R}^{N-n} .
$$

Thus it suffices to prove Theorem 1 when $N=n$.

Since the exponents $\alpha_{1}, \ldots, \alpha_{n+l}$ span $\mathbb{R}^{n}$, we may assume that the first $n$ are the standard unit basis vectors in $\mathbb{R}^{n}$, and thus $\varphi$ includes the coordinate exponentials $e^{z_{i}}$ for $i=1, \ldots, n$. Let $M:=\left(M_{0}, M_{1}, \ldots, M_{n}\right)$ be a list of positive numbers and set

$$
\Delta_{M}:=\left\{z \in \mathbb{R}^{n}: z_{i} \geq-M_{i}, i=1, \ldots, n \quad \text { and } \quad \sum_{i} z_{i} \leq M_{0}\right\},
$$

which is a nonempty simplex. We will use stratified Morse theory to bound the Betti numbers of $Y:=Z \cap \Delta_{M}$ when $M$ is general.

Theorem 4. For $M$ general, the sum of the Betti numbers of $Y$ is at most

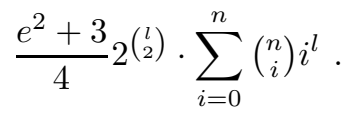

Proof of Theorem 1 . For any $r>0$, set

$$
Z_{r}:=\{z \in Z:\|z\|<r\} .
$$

By [4, Corollary 9.3.7], there is some $R>0$ such that if $r \geq R$, then $Z_{r}$ is a deformation retract of $Z$ and $Z_{R}$ is a deformation retract of $Z_{r}$.

Choose $M$ and $r>R$ so that $\Delta_{M}$ is sandwiched between the balls of radius $R$ and $r$ centered at the origin. Let $\rho: Z_{r} \rightarrow Z_{R}$ be the retraction. We have the maps

$$
Z_{R} \hookrightarrow Y=Z \cap \Delta_{M} \hookrightarrow Z_{r} \stackrel{\rho}{\longrightarrow} Z_{R},
$$

whose composition is the identity. The induced maps on the $i$ th homology groups,

$$
H_{i}\left(Z_{R}\right) \longrightarrow H_{i}(Y) \longrightarrow H_{i}\left(Z_{r}\right) \stackrel{\rho_{*}}{\longrightarrow} H_{i}\left(Z_{R}\right),
$$

have composition the identity. This gives the inequality

$$
\operatorname{dim} H_{i}(Y) \geq \operatorname{dim} H_{i}\left(Z_{R}\right)=\operatorname{dim} H_{i}(Z) .
$$

Summing over $i$ shows that Theorem 1 is a consequence of Theorem 4 .

Proof of Theorem 4, Given positive numbers $M=\left(M_{0}, M_{1}, \ldots, M_{n}\right)$, define affine hyperplanes in $\mathbb{R}^{n}$ by

$$
H_{0}:=\left\{z: \sum_{i} z_{i}=M_{0}\right\} \quad \text { and } \quad H_{i}:=\left\{z: z_{i}=-M_{i}\right\}, \quad \text { for } i=1, \ldots, n .
$$

For each proper subset $S \subset\{0, \ldots, n\}$, define an affine linear subspace

$$
H_{S}:=\bigcap_{i \in S} H_{i}
$$


Since each $M_{i}>0$, this has dimension $n-|S|$, and these subspaces are the affine linear subspaces supporting the faces of the simplex $\Delta_{M}$.

Choose $M$ generic so that for all $S$ the subspace $H_{S}$ meets $Z$ transversally. For each $S$, set $Z_{S}:=Z \cap H_{S}$. This is a smooth hypersurface in $H_{S}$ and therefore has dimension $n-|S|-1$. The boundary stratum $Y_{S}$ of $Y=Z \cap \Delta_{M}$ lying in the face supported by $H_{S}$ is an open subset of $Z_{S}$.

For a nonzero vector $u \in \mathbb{R}^{n}$, the directional derivative $D_{u} \varphi$ is

$$
\sum_{i=1}^{n+l}\left(u \cdot \alpha_{i}\right) c_{i} e^{z \cdot \alpha_{i}}
$$

which is an exponential sum having the same exponents as $\varphi$. Let $L_{u}$ be the linear function on $\mathbb{R}^{n}$ defined by $z \mapsto u \cdot z$.

The critical points of the function $L_{u}$ restricted to $Z$ are the zeroes of the system

$$
\varphi(z)=0 \quad \text { and } \quad D_{v} \varphi(z)=0 \text { for } v \in u^{\perp} .
$$

When $u$ is general and we choose a basis for $u^{\perp}$, this becomes a system of $n$ exponential sums in $n$ variables, all involving the same $n+l+1$ exponents. By the fewnomial bound in [3], the number of solutions is at most

$$
\frac{e^{2}+3}{4} 2^{\left(\begin{array}{l}
l \\
2
\end{array}\right)} n^{l}
$$

We use this to estimate the number of critical points of the function $L_{u}$ restricted to $Z_{S}$. The restriction of $\varphi$ to $H_{S}$ defines $Z_{S}$ as a hypersurface in $H_{S}$. We determine this restriction. If $i \in S$ with $i>0$, then we may use the equation $z_{i}=-M_{i}$ to eliminate the variable $z_{i}$ and the exponential $e^{z_{i}}$ from $\varphi$. If $0 \in S$, then we choose $j \notin S$ and use the equation $\sum_{i} z_{i}=M_{0}$ to eliminate $z_{j}$ from $\varphi$. Let $\varphi_{S}$ be the result of this elimination. It is an exponential sum in $n-|S|$ variables and its number of terms is at most

$$
\begin{aligned}
(n-|S|)+l+1 & \text { if } 0 \notin S, \\
(n-|S|)+(l+1)+1 & \text { if } 0 \in S .
\end{aligned}
$$

Thus if $u$ is a general vector in $\mathbb{R}^{n}$, then the number of critical points of the linear function $\left.L_{u}\right|_{H_{S}}$ on $Z_{S}$ is at most

$$
\begin{aligned}
& \frac{e^{2}+3}{4} 2^{\left(\begin{array}{l}
l \\
2
\end{array}\right)}(n-|S|)^{l} \quad \text { if } 0 \notin S, \\
& \frac{e^{2}+3}{4} 2^{\left(\begin{array}{c}
l+1 \\
2
\end{array}\right)}(n-|S|)^{l+1} \quad \text { if } 0 \in S .
\end{aligned}
$$

We use this estimate and stratified Morse theory to bound the Betti numbers of $Y$.

Let $u$ be a general vector in $\mathbb{R}^{n}$ such that $L_{u}$ is a Morse function for the stratified space $Y$. By Proposition 2, the sum of the Betti numbers of $Y$ is bounded by the number of critical points $p$ of $L_{u}$ for which $L_{u}$ achieves its minimum on the normal slice $N(p)$ at $p$. Since the strata $Y_{S}$ of $Y$ are open subsets of the manifolds $Z_{S}$, this number is bounded above by the number of such critical points of $L_{u}$ on the manifolds $Z_{S}$. We argue that we can alter $u$ so that no critical point in any $Z_{S}$ with $0 \in S$ contributes. That is, change $u$ so that for no critical point $p$ in a stratum $Z_{S}$ with $0 \in S$ is $p$ the local minimum of $L_{u}$ on the normal slice $N(p)$.

Suppose that $p$ is a critical point of $L_{u}$ on a stratum $Z_{S}$ with $0 \in S$. Then $p$ lies in $H_{0}$, and so the linear function $L_{(1, \ldots, 1)}$ restricted to the normal slice $N(p)$ at $p$ takes its maximum value $M_{0}$ at $p$. If we replace $u$ by $u+\lambda(1, \ldots, 1)$, we change $L_{u}$ on $H_{S}$ by the constant $\lambda M_{0}$ and $p$ will still be a critical point for $L_{u+\lambda(1, \ldots, 1)}$ on 
$Z_{S}$. If $\lambda$ is sufficiently large, then $L_{u+\lambda(1, \ldots, 1)}$ does not achieve its minimum value on $N(p)$ at $p$.

There are finitely many critical points $p$ of $L_{u}$ on strata $Z_{S}$ with $0 \in S$. Hence, there is a positive number $\lambda$ so that at each of these critical points $p, L_{u+\lambda(1, \ldots, 1)}$ does not achieve its minimum value on $N(p)$ at $p$. We may further choose $\lambda$ so that $L_{u+\lambda(1, \ldots, 1)}$ is a Morse function for the stratified space $Y$. Since only the critical points on strata $Z_{S}$ with $0 \notin S$ can contribute to the Betti numbers of $Y$, we see that its sum of Betti numbers is bounded above by

$$
\frac{e^{2}+3}{4} 2^{\left(\begin{array}{l}
l \\
2
\end{array}\right)} \sum_{S \subset\{1, \ldots, n\}}(n-|S|)^{l}=\frac{e^{2}+3}{4} 2^{\left(\begin{array}{l}
l \\
2
\end{array}\right)} \sum_{i=0}^{n}\left(\begin{array}{c}
n \\
i
\end{array}\right)(n-i)^{l} .
$$

Since $\left(\begin{array}{c}n \\ i\end{array}\right)=\left(\begin{array}{c}n \\ n-i\end{array}\right)$, we replace $i$ by $n-i$ to complete the proof of Theorem 4 ,

We remark that the idea of cutting a fewnomial variety with a monomial hypersurface, which preserves the fewnomial structure, is not ours, but may be found in papers of Rojas [11] and Perrucci [9, who used this to bound the number of connected components of a fewnomial variety.

We deduce the formula (2) from Theorem 11. Observe that

$$
\sum_{i=0}^{n}\left(\begin{array}{c}
n \\
i
\end{array}\right) i^{l}=n^{l} \sum_{i=0}^{n}\left(\begin{array}{c}
n \\
i
\end{array}\right)\left(\frac{i}{n}\right)^{l},
$$

and the sum is a decreasing function of $l$. When $l=1$, this sum is

$$
\sum_{i=0}^{n}\left(\begin{array}{c}
n \\
i
\end{array}\right) \frac{i}{n}=\sum_{i=1}^{n}\left(\begin{array}{c}
n-1 \\
i-1
\end{array}\right)=2^{n-1} \text {. }
$$

Substituting this into the formula of Theorem 11 gives (2).

\section{REFERENCES}

[1] R. Benedetti and J.-J. Risler, Real algebraic and semi-algebraic sets, Actualités Mathématiques, Hermann, Paris, 1990. MR1070358 (91j:14045)

[2] F. Bihan, J.M. Rojas, and F. Sottile, Sharpness of fewnomial bounds and the number of components of a fewnomial hypersurface, Algorithms in Algebraic Geometry (A. Dickenstein, F. Schreyer, and A. Sommese, eds.), IMA Volumes in Mathematics and its Applications, vol. 146, Springer, 2008, pp. 15-20. MR2397935

[3] F. Bihan and F. Sottile, New fewnomial upper bounds from Gale dual polynomial systems, Moscow Mathematical Journal 7 (2007), no. 3, 387-407. MR2343138 (2008g:13038)

[4] J. Bochnak, M. Coste, and M.-F. Roy, Real Algebraic Geometry, Ergebnisse der Mathematik und ihrer Grenzgebiete (3), vol. 36, Springer-Verlag, Berlin, 1998, revised translation of the 1987 French original. MR949442 (90b:14030)

[5] M. Goresky and R. MacPherson, Stratified Morse Theory, Ergebnisse der Mathematik und ihrer Grenzgebiete (3), vol. 14, Springer-Verlag, Berlin, 1988. MR932724 (90d:57039)

[6] A.G. Khovanskii, Fewnomials, Trans. of Math. Monographs, 88, Amer. Math. Soc, Providence, RI, 1991. MR1108621 (92h:14039)

[7] J. Milnor, On the Betti numbers of real varieties, Proc. Amer. Math. Soc. 15 (1964), 275-280. MR0161339 (28:4547)

[8] O. A. Oleinnik, Estimates of the Betti numbers of real algebraic hypersurfaces, Mat. Sbornik N.S. 28(70) (1951), 635-640. MR0044864(13:489b)

[9] D. Perrucci, Some bounds for the number of components of real zero sets of sparse polynomials, Discrete Comput. Geom. 34 (2005), no. 3, 475-495. MR2160050 (2006j:12001)

[10] J.-J. Risler, Les nombres de Betti des ensembles algébriques réels, Gaz. Math. No. 54 (1992), 57-58. 
[11] J. Maurice Rojas, Some speed-ups and speed limits for real algebraic geometry, J. Complexity 16 (2000), no. 3, 552-571. MR1787885 (2001k:14103)

[12] R. Thom, Sur l'homologie des variétés algébriques réelles, Differential and Combinatorial Topology (A Symposium in Honor of Marston Morse), Princeton Univ. Press, Princeton, NJ, 1965, pp. 255-265. MR0200942 (34:828)

Laboratoire de Mathématiques, Université de Savoie, 73376 Le Bourget-du-LaC Cedex, France

E-mail address: Frederic.Bihan@univ-savoie.fr

URL: http://www.lama.univ-savoie.fr/ bihan/

Department of Mathematics, Texas A\&M University, College Station, Texas 77843

E-mail address: sottile@math.tamu.edu

$U R L:$ http://www.math.tamu.edu/ ${ }^{\sim}$ sottile/ 